\title{
One-pot Three-component Synthesis of Novel Quinazoline-4-carboxylic Acid and Derivatives
}

\section{Aynı Ortamda Üç Bileşenli Yeni Kinazolin-4-karboksilik Asit ve Türevlerinin Sentezi}

\section{Derviş Gök}

Depart. of Chemistry and Chemical Processing Technologies, Kutahya Technical Sciences Vocational School, Dumlupinar University, Kutahya, Turkey.

\section{ABSTRACT}

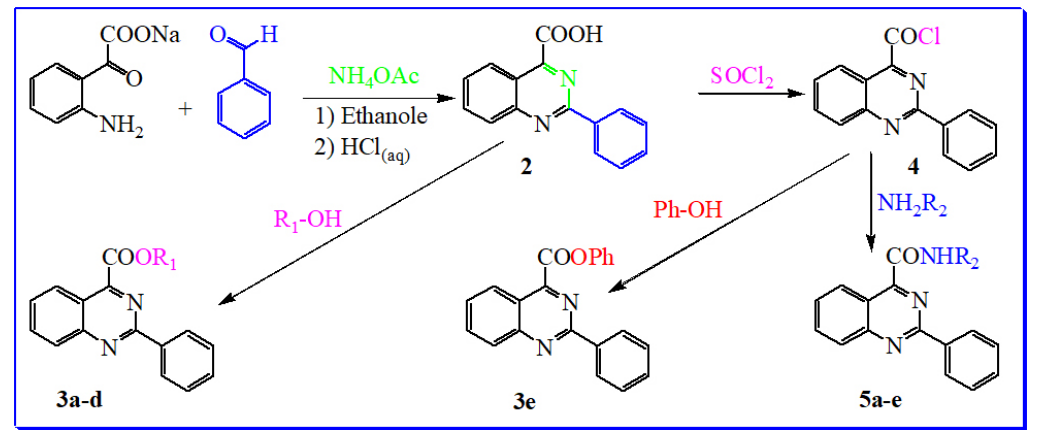

\begin{abstract}
-Phenyl-quinazoline-4-carboxylic acid (2) was synthesized from the one-pot three-component reaction of (2-amino- phenyl)-oxo-acetic acid sodium salt obtained from the hydrolysis of isatin with ammonium acetate and benzaldehyde. Some novel quinazoline-ester derivatives (3a-d) were then obtained by the reaction between 2 and various alcohols. Finally, quinazoline-amide derivatives (5a-e) were synthesized from the reaction of various amines and 2-phenyl-quinazoline-4carbonyl chloride (4), obtained by the reaction of compound 2 with $\mathrm{SOCl}_{2}$. The structures of synthesized compounds were clarified by ${ }^{1} \mathrm{H} N \mathrm{NM},{ }^{13} \mathrm{C}$ NMR, IR, mass spectrometry analysis methods.
\end{abstract}

\section{Key Words}

Quinazoline-4-carboxylic acid, isatin, ester, amide.

\section{öz}

monyum asetat ve benzaldehit ile isatinin hidrolizinden elde edilen (2-amino-fenil)-okso-asetik asit sodyum tuzunun aynı ortamda üç bileşenli reaksiyonundan 2-fenil-kinazolin-4-karboksilik asit (2) sentezlendi. Daha sonra 2 ve çeşitli alkoller arasındaki reaksiyonlarından bazı yeni kinazolin-ester türevleri (3a-d) elde edildi. Son olarak 2 bileşiği ile $\mathrm{SOCl}_{2}$ 'nin reaksiyonundan elde edilen 2 -fenil-kinazolin-4-karbonil klorür ve çeşitli aminlerin reaksiyonundan kinazolin-amit türevleri (5a-e) sentezlendi. Sentezlenen bileşiklerin yapıları ${ }^{1} \mathrm{H} N M R,{ }^{13} \mathrm{C} N M R, I R$, kütle spektrometri analiz metotları tarafından doğrulandı.

\section{Anahtar Kelimeler}

Kinazolin-4-karboksilik asit, isatin, ester, amit.

Article History: Nov 18, 2018; Revised Dec, 23 2019; Accepted: Jan 29, 2020; Available Online: May 3, 2020. DOI: https://doi.org/10.15671/hibc.736847

Correspondence to: D. Gök, Department of Chemistry and Chemical Processing Technologies, Kutahya Technical Sciences Vocational School, Dumlupinar University, Kutahya, Turkey.

E-Mail: dervis.gok@dpu.edu.tr 


\section{INTRODUCTION}

( $)$ uinazoline and its derivatives are among the most important $\mathrm{N}$-heterocyclic structures. The compounds containing the quinazoline ring both form the core structure of many natural products and exhibit useful biological activities such as anticancer [1], antimicrobial [2], anti-oxidant [3], antimalarial [4], anti-inflammatory [5], antiplasmodial [6], antiviral [7], anti-HIV [8], anticonvulsant [9], and anti-diabetic [10]. Bicyclic quinazoline ring constitutes the core structure of some drug molecules such as icotinib [11], lapatinib [12], and prazosin [13]. In addition, some compounds based on quinazoline are used as anticancer drugs such as erlotinib [14] and gefitinib [15].

Studies have shown that some quinazoline derivatives are a novel chiral fluorescent tubulin binding agent with highly potent antiproliferative properties against human cancer cells [16]. Furthermore, quinazoline derivatives are known to exhibit aurora a kinase inhibitor effect [17] and cytotoxic activity against human cancer cell [18]. Some of them also exhibit the inhibitory effect of epidermal growth factor receptor tyrosine kinase [19] and act as anti-cancer DNA binding alkaloids [20]. In the study of Seo et al., 3,4-dihydroquinazoline derivatives have been found to perform excellent T-type calcium channel blocking activity [21].

The above studies clearly demonstrate the importance of quinazoline derivatives. Their increasing importance has led to the development of different methods for their synthesis. Some reactions for their synthesis are aerobic oxidative coupling reactions of $\mathrm{N}$-arylamidines with benzyl alcohol or aromatic aldehydes in air [22], cyclization and aromatization reactions of amidine hydrochlorides with methyl 2-halobenzoate, 2-halophenylketone, or 2-halobenzaldehyde derivatives [23], and cyclization reactions benzamidines and 2-bromobenzylbromides [24].

Different procedures have been developed for the synthesis of quinazolines such as photochemical methods [25], copper-catalyzed syntheses [26], reactions of urea maltose and ammonium chloride mixture [27], the use of microwave irradiation [28], and tandem reactions from benzylic amines and 2-aminobenzophenones [29].
The isatin compound was used as the starting compound in many reactions. It was also the starting compound of our reactions. It was hydrolyzed in alkaline medium to form (2-amino-phenyl)-oxo-acetic acid sodium salt. Quinazoline-4-carboxylic acid derivative was synthesized from one-pot three-component reaction of (2-amino-phenyl)-oxo-acetic acid sodium salt with ammonium acetate and benzaldehyde compounds. The literature review showed that the carboxyl group bound to the quinazoline ring was absent and that it was synthesized for the first time in our study. This paper reports the synthesis and characterization of some new quinazoline-4-carboxylic acid derivatives.

Firstly, quinazoline-4-carboxylic acid sodium salt was synthesized via reactions between readily available benzaldehyde (2-amino-phenyl)-oxo-acetic acid sodium salt and ammonium acetate in ethanol under mild conditions without catalyst. Two mechanisms are proposed for this reaction where the quinazoline ring is formed [30]. The proposed mechanisms are shown in scheme 1.

In the first reaction mechanism (path a), the condensation reaction of the aldehyde and 2-aminobenzophenone gives aldimine compound. The condensation reaction of ammonium acetate with this intermediate then gives diimine compound. The diimine compound is converted to the quinazoline compound by the cyclization reaction followed by aromatization reactions. In the other mechanism (path b), the condensation reaction of the aldehyde and ammonium acetate gives aldimine compound. Subsequent cyclization and aromatization reactions of 2-aminobenzophenone with this intermediate aldimine are converted to the quinazoline compound. The quinazoline compound formed in these two reaction mechanisms is obtained by air oxidation of the 1,2-dihydroquinazoline compound.

As seen from the reaction mechanism, one of the two nitrogen atoms found in the quinazoline ring comes from the amine group of the hydrolysis isatin compound and the other is the reactant ammonium acetate. Since we used the sodium salt of the hydrolysis isatin compound in the quinazoline reaction, we obtained the quinazoline compound as the carboxylic acid salt (1). Then, when we acidified this carboxylic acid salt with $\mathrm{HCl}_{(\mathrm{aq})}$, we converted to 2-phenyl-quinazoline-4carboxylic acid (2). The presence of the carboxyl group bound to the quinazoline ring then allowed the synthe- 


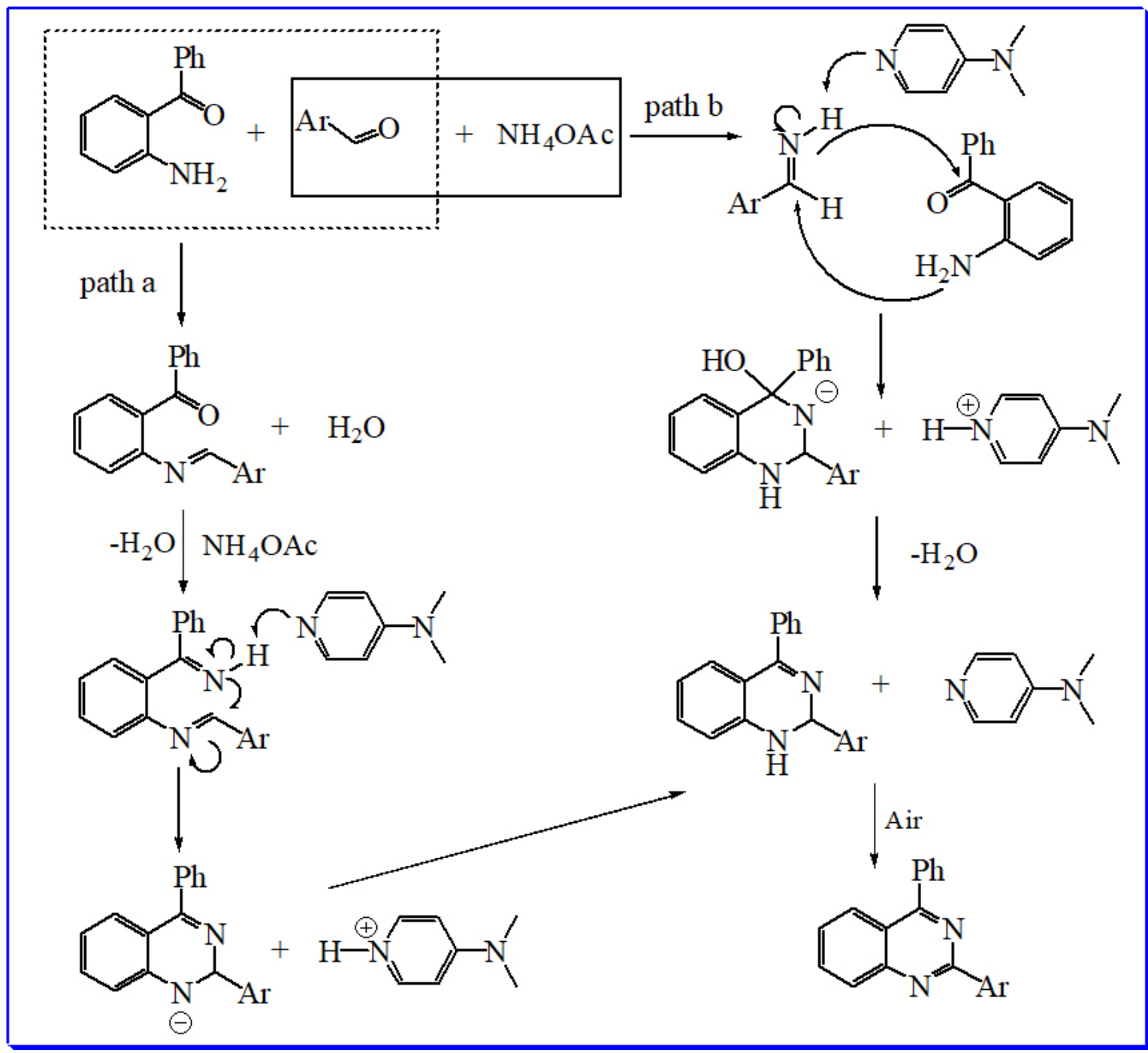

Scheme 1. Proposed mechanisms for the synthesis of 1,2-dihydroquinazolines and quinazolines with DMAP-catalyzed [30].

sis of carboxylic acid derivatives. The carboxyl group in the quinazoline ring was easily converted to the ester (3a-e) acid chloride (4) and amide (5a-e) derivatives. The synthesis of compounds is shown in scheme 2.

\section{MATERIALS and METHODS}

\section{Chemicals and Instruments}

The chemicals used in the synthesis of all new compounds were purchased from Merck and Aldrich Chemical Company. All chemicals and solvents were of spectroscopic reagent grade. The reactions were monitored by thin-layer chromatography (TLC) using aluminum sheets coated with silica gel $60_{\mathrm{F} 254}$ (Merck) Purity of the synthesized compounds was confirmed by TLC in the same way. Spots were detected by their absorption under UV light $(\lambda=254 \mathrm{~nm})$. Melting points were measu- red on a Stuart SMP30 apparatus. The IR data (Agilent Technologies Inc., Santa Clara, CA) were recorded on a Bruker Vertex 70 Sample compartment spectrometer using potassium bromide pellets. ${ }^{1} \mathrm{H}$ NMR and ${ }^{13} \mathrm{C}$ NMR spectra were recorded on a Bruker AVANS $300 \mathrm{MHz}$ spectrometer operating at $300 \mathrm{MHz}$ and $75 \mathrm{MHz}$ for ${ }^{1} \mathrm{H}$ and ${ }^{13} \mathrm{C}$ nuclei, respectively, in deuterochloroform and dimethyl sulphoxide- $d_{6}$ with tetramethylsilane as internal standard. Shifts were given in ppm, coupling constant $(J)$ values were presented in hertz $(\mathrm{Hz})$, and the abbreviations were as follows: $s$ (singlet), $d$ (doublet), $t$ (triplet), and $\mathrm{m}$ (multiplet). The mass analyses were performed on an Agilent Technologies 6530 Accurate-Mass Q-TOF LC/MS at the advanced technology research center of Dumlupinar University (ILTEM). 
3a: $\mathrm{R}_{1}=\mathrm{CH}_{3}$

3b: $\mathrm{R}_{1}=\mathrm{CH}_{2} \mathrm{CH}_{3}$

3c: $\mathrm{R}_{1}=\mathrm{CH}\left(\mathrm{CH}_{3}\right)_{2}$

3d: $\mathrm{R}_{1}=\left(\mathrm{CH}_{2}\right)_{3} \mathrm{CH}_{3}$<smiles>CCOC(=O)c1nc(-c2ccccc2)nc2ccccc12</smiles>

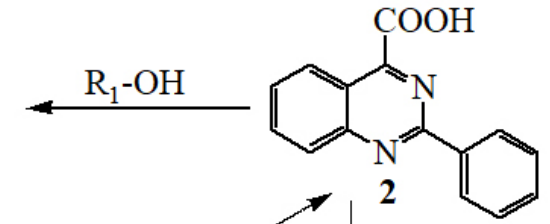<smiles>Nc1ccccc1C(=O)O[Na]</smiles><smiles>CCCC(N)=O</smiles>

Ethanol

Reflux

5a: $\mathrm{R}_{2}=\mathrm{H}$

5b: $\mathrm{R}_{2}=\left(\mathrm{CH}_{2}\right)_{2} \mathrm{CH}_{3}$

5c: $\mathrm{R}_{2}=\mathrm{CH}\left(\mathrm{CH}_{3}\right)_{2}$

5d: $\mathrm{R}_{2}=\mathrm{Ph}$

5e: $\mathrm{R}_{2}=\longrightarrow-\mathrm{SO}_{2} \mathrm{NH}_{2}$

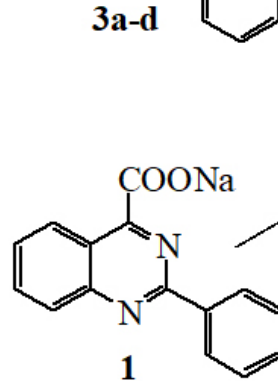
$\mathrm{SOCl}_{2}$

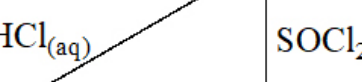

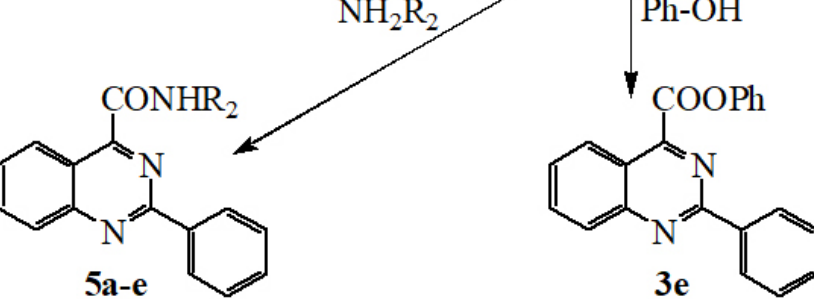

Scheme 2. Synthesis of novel quinazoline-4-carboxylic acid derivatives.

\section{Synthesis}

\section{2-Phenyl-quinazoline-4-carboxylic acid sodium salt}

(1). A mixture of ammonium acetate $(0.154 \mathrm{~g}, 2 \mathrm{mmol})$ and benzaldehyde $(0.106 \mathrm{~g}, 1 \mathrm{mmol})$ were added to a solution of (2-amino-phenyl)-oxo-acetic acid sodium salt $(0.187 \mathrm{~g}, 1 \mathrm{mmol})$ in ethanol $(10 \mathrm{~mL})$ at room temperature. The mixture was stirred and heated to reflux for $24 \mathrm{~h}$. The formed precipitate was filtered while it was still hot. The product was crystallized from water and dried in vacuo at $70^{\circ} \mathrm{C}$. Yield: $0.08 \mathrm{~g}(29 \%) ; \mathrm{mp}$ above $350^{\circ} \mathrm{C}$; IR $\left(v, \mathrm{~cm}^{-1}\right)$ : $3061(\mathrm{Ar} \mathrm{CH}), 1650(\mathrm{C}=\mathrm{O})$, 1587$1459(\mathrm{C}=\mathrm{C}$ and $\mathrm{C}=\mathrm{N})$; ${ }^{1} \mathrm{H}$ NMR (300 MHz, DMSO-d $\left.{ }_{6}\right) \delta$ (ppm): 8.55-8.52 (m, 2H, Ar-H), $8.13(\mathrm{~d}, \mathrm{~J}=8.25 \mathrm{~Hz}, 1 \mathrm{H}$ Ar-H), 7.97-7.87 (m, 2H, Ar-H), 7.63-7.53 (m, 4H, other Ar$\mathrm{H}) ;{ }^{13} \mathrm{C}$ NMR (75 MHz, DMSO-d 6 ) $\delta$ (ppm): 170.54 (COONa), 169.49, 159.82, 150.88, 138.42, 134.27, 130.93, $129.02,128.54,128.35,128.23,127.23,119.62$; HRMS (QTOF-ESI): $\mathrm{m} / \mathrm{z}$ calcd for $\mathrm{C}_{15} \mathrm{H}_{9} \mathrm{~N}_{2} \mathrm{NaO}_{2}: 272.0562$; found: $273.0637[\mathrm{M}+1]^{+}$

2. 2-Phenyl-quinazoline-4-carboxylic acid (2). Compound 1 (0.272 g, $1 \mathrm{mmol}$ ) was added to distilled water $(10 \mathrm{~mL})$. The obtained solution was heated to $90^{\circ} \mathrm{C}$ and slowly acidified with $2 \mathrm{~N} \mathrm{HCl}_{(\text {aq) }}$ till reaching $\mathrm{pH} 1$. The resulting mixture was kept at $5^{\circ} \mathrm{C}$ overnight. Then, the precipitate was filtered off and washed with water $(3 \mathrm{x}$
$15 \mathrm{~mL})$. The product was crystallized from toluene and dried in vacuo at $70^{\circ} \mathrm{C}$. Yield: $0.21 \mathrm{~g}(84 \%) ; \mathrm{mp} 153^{\circ} \mathrm{C}$; IR $\left(v, \mathrm{~cm}^{-1}\right)$ : 3500-2500 (COOH), $3056(\mathrm{Ar} \mathrm{CH}), 1713(\mathrm{C}=\mathrm{O}$, acid), 1614-1442 (C=C and $\mathrm{C}=\mathrm{N}) ;{ }^{1} \mathrm{H}$ NMR (300 MHz, DMSO-d $\left.{ }_{6}\right) \delta(p p m): 14.42(\mathrm{br}, \mathrm{s}, 1 \mathrm{H}, \mathrm{COOH}), 8.60-8.57$ (m, 2H, Ar-H), 8.42 (d, J=8.32 Hz, 1H, Ar-H), 8.19-8.09 (m, $2 \mathrm{H}, \mathrm{Ar}-\mathrm{H}), 7.82(\mathrm{t}, \mathrm{J}=7.60,1 \mathrm{H}, \mathrm{Ar}-\mathrm{H}), 7.62-7.59(\mathrm{~m}, 3 \mathrm{H}$, other $\mathrm{Ar}-\mathrm{H}) ;{ }^{13} \mathrm{C}$ NMR (75 MHz, DMSO-d 6 ) $\delta$ (ppm): 166.79 (C=O, acid), 159.88, 159.47, 151.85, 137.18, 135.67, 131.60, 129.26, 129.18, 129.09, 128.66, 126.42, 119.77; HRMS (QTOF-ESI): $\mathrm{m} / \mathrm{z}$ calcd for $\mathrm{C}_{15} \mathrm{H}_{10} \mathrm{~N}_{2} \mathrm{O}_{2}: 250.0742$ found: $251.0816[\mathrm{M}+1]^{+}$.

3. 2-Phenyl-quinazoline-4-carboxylic acid methyl ester (3a). $\mathrm{H}_{2} \mathrm{SO}_{4}(95-97 \%, 0.5 \mathrm{~mL}$ ) was added to a solution of $2(0.25 \mathrm{~g}, 1 \mathrm{mmol})$ in methanol $(20 \mathrm{~mL})$ at room temperature, and the mixture was stirred and heated to reflux for $6 \mathrm{~h}$. The resulting mixture was filtered while it was still hot. The solvents were removed on a rotary evaporator at $40^{\circ} \mathrm{C}$. The residue was washed with water $(3 \times 15 \mathrm{~mL})$. The product was crystallized from methanol-water mixture and dried in vacuo at $40^{\circ} \mathrm{C}$. Yield: 0.19 g (72\%); mp $109^{\circ} \mathrm{C}$; IR ( $\left.v, \mathrm{~cm}^{-1}\right)$ : 3093 (Ar CH), 2951 (aliphatic $\mathrm{CH}), 1726(\mathrm{C}=\mathrm{O}$, ester), 1617-1457 ( $\mathrm{C}=\mathrm{C}$ and $\mathrm{C}=\mathrm{N})$; ${ }^{1} \mathrm{H}$ NMR (300 MHz, $\mathrm{CDCl}_{3}$ ) $\delta$ (ppm): 8.66-8.63 (m, $2 \mathrm{H}$, Ar-H), 8.52 (d, J= $8.51 \mathrm{~Hz}, 1 \mathrm{H}, \operatorname{Ar}-\mathrm{H}), 8.18(\mathrm{~d}, \mathrm{~J}=8.58 \mathrm{~Hz}$, 
$1 \mathrm{H}, \mathrm{Ar}-\mathrm{H}), 7.96(\mathrm{t}, \mathrm{J}=8.36,1 \mathrm{H}, \mathrm{Ar}-\mathrm{H}), 7.68(\mathrm{t}, \mathrm{J}=8.22,1 \mathrm{H}$, Ar-H), 7.57-7.53 (m, 3H, other Ar-H), $4.16\left(\mathrm{~s}, 3 \mathrm{H}, \mathrm{OC}_{3}\right)$; $\left.{ }^{13} \mathrm{C} \mathrm{NMR} \mathrm{(75} \mathrm{MHz}, \mathrm{CDCl}_{3}\right) \delta$ (ppm): 165.59 ( $\mathrm{C}=\mathrm{O}$, ester), $160.18,157.08,152.38,137.23,134.50,130.98,129.15$, 128.73, 128.70, 128.33, 125.90, 120.46, $53.27\left(\mathrm{OCH}_{3}\right)$; HRMS (QTOF-ESI): $\mathrm{m} / \mathrm{z}$ calcd for $\mathrm{C}_{16} \mathrm{H}_{12} \mathrm{~N}_{2} \mathrm{O}_{2}:$ 264.0899; found: $265.0976[\mathrm{M}+1]^{+}$.

\section{2-Phenyl-quinazoline-4-carboxylic acid ethyl ester}

(3b). $\mathrm{H}_{2} \mathrm{SO}_{4}(95-97 \%, 0.5 \mathrm{~mL}$ ) was added to a solution of $2(0.25 \mathrm{~g}, 1 \mathrm{mmol})$ in ethanol $(25 \mathrm{~mL})$ at room temperature, and the mixture was stirred and heated to reflux for $5 \mathrm{~h}$. The resulting mixture was filtered while it was still hot. The solvents were removed on a rotary evaporator at $40^{\circ} \mathrm{C}$. The residue was washed with water $(3$ $\times 15 \mathrm{~mL})$. The obtained oily product was dried in vacuo at $40^{\circ} \mathrm{C}$. Yield: $0.17 \mathrm{~g}(61 \%) ; \mathrm{mp}$ oily; $\mathrm{IR}\left(\mathrm{v}, \mathrm{cm}^{-1}\right): 3064$ ( $\mathrm{Ar} \mathrm{CH}), 2981$ (aliphatic $\mathrm{CH}), 1727(\mathrm{C}=\mathrm{O}$, ester), 1616$1458(\mathrm{C}=\mathrm{C}$ and $\mathrm{C}=\mathrm{N}) ;{ }^{1} \mathrm{H} \mathrm{NMR}\left(300 \mathrm{MHz}, \mathrm{CDCl}_{3}\right) \delta(\mathrm{ppm})$ : 8.67-8.63 (m, 2H, Ar-H), $8.46(\mathrm{~d}, \mathrm{~J}=8.47 \mathrm{~Hz}, 1 \mathrm{H}, \mathrm{Ar}-\mathrm{H})$, $8.15(\mathrm{~d}, \mathrm{~J}=8.52 \mathrm{~Hz}, 1 \mathrm{H}, \mathrm{Ar}-\mathrm{H}), 7.94(\mathrm{t}, \mathrm{J}=7.70,1 \mathrm{H}, \mathrm{Ar}-\mathrm{H})$, $7.65(t, J=7.77,1 \mathrm{H}, \mathrm{Ar}-\mathrm{H}), 7.57-7.52(\mathrm{~m}, 3 \mathrm{H}$, other $\mathrm{Ar}-\mathrm{H})$, 4.63 (q, J=7.14 Hz, 2H, OC$\left.{ }_{2}\right), 1.54$ (t, J=7.10 Hz, 3H, $\left.\underline{C}_{3}\right)$; $\left.{ }^{13} \mathrm{C} \mathrm{NMR} \mathrm{(75} \mathrm{MHz}, \mathrm{CDCl}_{3}\right) \delta$ (ppm): 164.34 ( $\mathrm{C}=\mathrm{O}$, ester), 159.24, 156.76, 151.44, 136.46, 133.48, 130.04, 128.27, $127.84,127.75,127.28,124.91,119.44,61.68\left(\mathrm{OCH}_{2}\right)$ $13.44\left(\mathrm{CH}_{3}\right)$; HRMS (QTOF-ESI): $\mathrm{m} / \mathrm{z}$ calcd for $\mathrm{C}_{17} \mathrm{H}_{14} \mathrm{~N}_{2} \mathrm{O}_{2}$ : 278.1055; found: $278.1664[\mathrm{M}]^{+}$.

\section{2-Phenyl-quinazoline-4-carboxylic acid isopropyl} ester (3c). $\mathrm{H}_{2} \mathrm{SO}_{4}(95-97 \%, 0.5 \mathrm{~mL})$ was added to a solution of $2(0.25 \mathrm{~g}, 1 \mathrm{mmol})$ in isopropanol $(20 \mathrm{~mL})$ at room temperature, and the mixture was stirred and heated to reflux for $5 \mathrm{~h}$. The resulting mixture was filtered while it was still hot. The solvents were removed on a rotary evaporator at $40^{\circ} \mathrm{C}$. The residue was washed with water $(3 \times 15 \mathrm{~mL})$. The obtained oily product was dried in vacuo at $40^{\circ} \mathrm{C}$. Yield: $0.17 \mathrm{~g}(58 \%) ; \mathrm{mp}$ oily; IR $(v, \mathrm{~cm}$ 1): 3064 ( $\mathrm{Ar} \mathrm{CH}), 2981$ (aliphatic $\mathrm{CH}), 1723$ ( $\mathrm{C}=\mathrm{O}$, ester), 1616-1457 ( $\mathrm{C}=\mathrm{C}$ and $\mathrm{C}=\mathrm{N}) ;{ }^{1} \mathrm{H}$ NMR $\left(300 \mathrm{MHz}, \mathrm{CDCl}_{3}\right) \delta$ (ppm): 8.66-8.64 (m, 2H, Ar-H), 8.39 (d, J= $8.44 \mathrm{~Hz}, 1 \mathrm{H}$, Ar-H), $8.14(\mathrm{~d}, J=8.49 \mathrm{~Hz}, 1 \mathrm{H}, \mathrm{Ar}-\mathrm{H}), 7.93(\mathrm{t}, \mathrm{J}=8.33,1 \mathrm{H}$, Ar-H), $7.65(\mathrm{t}, \mathrm{J}=7.35,1 \mathrm{H}, \mathrm{Ar}-\mathrm{H}), 7.57-7.51(\mathrm{~m}, 3 \mathrm{H}$, other Ar-H), 6.28 (heptet, J=6.28 Hz, 1H, OCH), 1.53 (d, J=6.25 $\left.\mathrm{Hz}, 6 \mathrm{H}, 2 \mathrm{CH}_{3}\right) ;{ }^{13} \mathrm{C} \mathrm{NMR}\left(75 \mathrm{MHz}, \mathrm{CDCl}_{3}\right) \delta$ (ppm): 164.89 ( $\mathrm{C}=\mathrm{O}$, ester), 160.18, 158.32, 152.27, 137.39, 134.33, 130.91, 129.19, 128.75, 128.62, 128.09, 125.72, 120.23, $70.65(\mathrm{OCH}), 21.94\left(\mathrm{CH}_{3}\right)$; HRMS (QTOF-ESI): $\mathrm{m} / \mathrm{z}$ calcd for $\mathrm{C}_{18} \mathrm{H}_{16} \mathrm{~N}_{2} \mathrm{O}_{2}:$ :292.1212; found: $292.2651[\mathrm{M}]^{+}$.

\section{2-Phenyl-quinazoline-4-carboxylic acid butyl ester} (3d). $\mathrm{H}_{2} \mathrm{SO}_{4}(95-97 \%, 0.5 \mathrm{~mL})$ was added to a solution of $2(0.25 \mathrm{~g}, 1 \mathrm{mmol})$ in $n$-butanol $(30 \mathrm{~mL})$ at room temperature, and the mixture was stirred and heated to reflux for $5 \mathrm{~h}$. The resulting mixture was filtered while it was still hot. The solvents were removed on a rotary evaporator at $40^{\circ} \mathrm{C}$. The residue was washed with water $(3$ $x 15 \mathrm{~mL})$. The obtained oily product was dried in vacuo at $40^{\circ} \mathrm{C}$. Yield: $0.16 \mathrm{~g}(52 \%) ; \mathrm{mp}$ oily; IR $\left(v, \mathrm{~cm}^{-1}\right): 3064$ (Ar CH), 2960 (aliphatic CH), $1726(\mathrm{C}=\mathrm{O}$, ester), 1616$1458(\mathrm{C}=\mathrm{C}$ and $\mathrm{C}=\mathrm{N}) ;{ }^{1} \mathrm{H} \mathrm{NMR}\left(300 \mathrm{MHz}, \mathrm{CDCl}_{3}\right) \delta(\mathrm{ppm})$ : 8.65-8.62 (m, 2H, Ar-H), $8.44(\mathrm{~d}, \mathrm{~J}=8.47 \mathrm{~Hz}, 1 \mathrm{H}, \mathrm{Ar}-\mathrm{H})$, $8.15(\mathrm{~d}, \mathrm{~J}=8.55 \mathrm{~Hz}, 1 \mathrm{H}, \mathrm{Ar}-\mathrm{H}), 7.94(\mathrm{t}, \mathrm{J}=7.72,1 \mathrm{H}, \mathrm{Ar}-\mathrm{H})$, $7.65(\mathrm{t}, \mathrm{J}=7.76,1 \mathrm{H}, \mathrm{Ar}-\mathrm{H}), 7.57-7.51(\mathrm{~m}, 3 \mathrm{H}$, other Ar-H), $4.57\left(\mathrm{t}, \mathrm{J}=6.72 \mathrm{~Hz}, 2 \mathrm{H}, \mathrm{OC}_{2}\right.$ ), 1.89 (pentet, J=7.88 Hz, 2H, $\mathrm{OCH}_{2} \mathrm{CH}_{2}$ ), 1.56 (hextet, J=7.54 Hz, 2H, $\mathrm{CH}_{2} \mathrm{CH}_{3}$ ), 1.03 (t, $\left.\mathrm{J}=7.33 \mathrm{~Hz}, 3 \mathrm{H}, \mathrm{CH}_{3}\right) ;{ }^{13} \mathrm{C} \mathrm{NMR}\left(75 \mathrm{MHz}, \mathrm{CDCl}_{3}\right) \delta(\mathrm{ppm})$ : 165.34 ( $\mathrm{C}=\mathrm{O}$, ester), 160.13, 157.72, 152.34, 137.38, $134.33,130.92,129.18,128.73,128.64,128.14,125.80$, 120.36, $66.35\left(\mathrm{OCH}_{2}\right), 30.67\left(\mathrm{OCH}_{2} \mathrm{CH}_{2}\right), 19.25\left(\mathrm{CH}_{2} \mathrm{CH}_{3}\right)$, $13.80\left(\mathrm{CH}_{3}\right)$; HRMS (QTOF-ESI): $\mathrm{m} / z$ calcd for $\mathrm{C}_{19} \mathrm{H}_{18} \mathrm{~N}_{2} \mathrm{O}_{2}$ : 306.1368; found: $307.1469[\mathrm{M}+1]^{+}$.

7. 2-Phenyl-quinazoline-4-carbonyl chloride (4). A mixture of $2(0.25 \mathrm{~g}, 1 \mathrm{mmol})$ and $\mathrm{SOCl}_{2}(\% 95 ; 5 \mathrm{~mL})$ were heated in an oil bath $\left(80^{\circ} \mathrm{C}\right)$ for $6 \mathrm{~h}$. The solvents were removed on a rotary evaporator at $50^{\circ} \mathrm{C}$. The residue was washed with ether $(3 \times 15 \mathrm{~mL})$. The product was crystallized from toluene-hexane mixture and dried in vacuo at $70^{\circ} \mathrm{C}$. Yield: $0.20 \mathrm{~g}(75 \%) ; \mathrm{mp} 149^{\circ} \mathrm{C}$; IR $\left(v, \mathrm{~cm}^{-1}\right): 3065(\mathrm{ArCH}), 1711$ (C=O, carbonyl), 1624-1457 $(\mathrm{C}=\mathrm{C}$ and $\mathrm{C}=\mathrm{N}) ;{ }^{1} \mathrm{H}$ NMR $\left(300 \mathrm{MHz}, \mathrm{CDCl}_{3}\right) \delta(\mathrm{ppm}): 9.37$ (d, J=8.63 Hz, 1H, Ar-H), 8.64-8.60 (m, 2H, Ar-H), 8.39 (d, J=8.42 Hz, 1H, Ar-H), 8.07 (t, J=7.83, 1H, Ar-H), 7.80 $(\mathrm{t}, \mathrm{J}=8.29,1 \mathrm{H}, \mathrm{Ar}-\mathrm{H})$, 7.61-7.59 (m, 3H, other $\mathrm{Ar}-\mathrm{H}) ;{ }^{13} \mathrm{C}$ NMR (75 MHz, DMSO-d $) \delta$ (ppm): 166.70 (C=O, carbonyl), 160.04, 159.46, 151.79, 137.16, 135.75, 131.63, 129.29, 129.24, 129.07, 128.65, 126.40, 119.72; HRMS (QTOF-ESI): $\mathrm{m} / \mathrm{z}$ calcd for $\mathrm{C}_{15} \mathrm{H}_{9} \mathrm{CIN} \mathrm{O}_{2} \mathrm{O}: 268.0403$; found: $269.0955[\mathrm{M}+1]^{+}$.

\section{2-Phenyl-quinazoline-4-carboxylic acid phenyl es-} ter (3e). A mixture of phenol $(0.094 \mathrm{~g}, 1 \mathrm{mmol})$ and triethylamine $(0.10 \mathrm{~g}, 1 \mathrm{mmol})$ were added to a solution of $4(0.268 \mathrm{~g}, 1 \mathrm{mmol})$ in toluene $(30 \mathrm{~mL})$ at room temperature. The mixture was stirred and heated to reflux for $5 \mathrm{~h}$. The reaction mixture was filtered while it was still hot. The solvents were removed on a rotary evaporator at $40^{\circ} \mathrm{C}$. The residue was washed with ether $(3 \times 15 \mathrm{~mL})$. The product was crystallized from toluene and dried in 
vacuo at $40^{\circ} \mathrm{C}$. Yield: $0.14 \mathrm{~g}(43 \%) ; \mathrm{mp} 121^{\circ} \mathrm{C}$; IR ( $v, \mathrm{~cm}$ $\left.{ }^{1}\right)$ : 3095 ( $\mathrm{ArCH}$ ), 1749 ( $\mathrm{C}=\mathrm{O}$, ester), 1615-1455 ( $\mathrm{C}=\mathrm{C}$ and $\mathrm{C}=\mathrm{N}) ;{ }^{1} \mathrm{H}$ NMR $\left(300 \mathrm{MHz}, \mathrm{CDCl}_{3}\right) \delta$ (ppm): 8.73-8.69 (m, $2 \mathrm{H}, \mathrm{Ar}-\mathrm{H}), 8.64(\mathrm{~d}, \mathrm{~J}=8.51 \mathrm{~Hz}, 1 \mathrm{H}, \mathrm{Ar}-\mathrm{H}), 8.20(\mathrm{~d}, \mathrm{~J}=8.53$ $\mathrm{Hz}, 1 \mathrm{H}, \mathrm{Ar}-\mathrm{H}), 7.98(\mathrm{t}, \mathrm{J}=7.75,1 \mathrm{H}, \mathrm{Ar}-\mathrm{H}), 7.70(\mathrm{t}, \mathrm{J}=7.64,1 \mathrm{H}$ Ar-H), 7.60-7.33 (m, 8H, other Ar-H); ${ }^{13} \mathrm{C} N M R(75 \mathrm{MHz}$, $\left.\mathrm{CDCl}_{3}\right) \delta(\mathrm{ppm}): 163.67$ (C=O, ester), 160.23, 156.22, $152.69,150.73,137.25,134.57,131.09,129.76,129.38$ 128.80, 128.75, 128.56, 126.57, 125.74, 121.62, 120.74; HRMS (QTOF-ESI): $\mathrm{m} / \mathrm{z}$ calcd for $\mathrm{C}_{21} \mathrm{H}_{14} \mathrm{~N}_{2} \mathrm{O}_{2}: 326.1055$; found: $327.1138[M+1]^{+}$.

\section{2-Phenyl-quinazoline-4-carboxylic acid amide (5a).} A mixture of 4 (0.268 g, $1 \mathrm{mmol})$ and THF (30 mL) were cooled to $0^{\circ} \mathrm{C}$. The obtained solution was slowly added to ammonium hydroxide solution $(0.15 \mathrm{~mL}, 2 \mathrm{mmol})$ at $0^{\circ} \mathrm{C}$, stirred, and kept at this temperature for $2 \mathrm{~h}$. The reaction mixture was continued and stirred at room temperature for an additional $2 \mathrm{~h}$. The obtained solution was filtered. The solvents were removed on a rotary evaporator at $40^{\circ} \mathrm{C}$. The residue was washed with ether $(3 \times 15 \mathrm{~mL})$. The product was crystallized from tolueneTHF mixture and dried in vacuo at $70^{\circ} \mathrm{C}$. Yield: $0.19 \mathrm{~g}$ (76\%); mp $198^{\circ} \mathrm{C} ; \mathrm{IR}\left(v, \mathrm{~cm}^{-1}\right): 3178(\mathrm{NH}), 3069(\mathrm{Ar} \mathrm{CH})$ 1710 ( $\mathrm{C}=\mathrm{O}$, amide), 1615-1488 ( $\mathrm{C}=\mathrm{C}$ and $\mathrm{C}=\mathrm{N}) ;{ }^{1} \mathrm{H}$ NMR

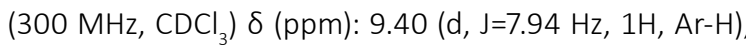
8.61-8.58 (m, 2H, Ar-H), 8.15 (d, J=8.49 Hz, 1H, Ar-H), $7.95(t, J=7.73,1 H, A r-H), 7.69(t, J=7.68,1 H, A r-H), 7.59$ $7.55(\mathrm{~m}, 3 \mathrm{H}$, other $\mathrm{Ar}-\mathrm{H}), 5.83\left(\mathrm{br}, \mathrm{s}, 2 \mathrm{H}, \mathrm{NH}_{2}\right) ;{ }^{13} \mathrm{C} \mathrm{NMR}$ (75 MHz, CDCl$)_{3}$ ) (ppm): 167.24 (C=O, amide), 154.90, 153.37, 137.16, 134.53, 130.96, 128.93, 128.74, 128.61, 128.40, 127.50, 121.09; HRMS (QTOF-ESI): $\mathrm{m} / \mathrm{z}$ calcd for $\mathrm{C}_{15} \mathrm{H}_{11} \mathrm{~N}_{3} \mathrm{O}: 249.0902$; found: $250.0962[\mathrm{M}+1]^{+}$.

\section{2-Phenyl-quinazoline-4-carboxylic acid propyla-} mide (5b). $n$-Propylamine (0.118 g, $2 \mathrm{mmol}$ ) was added to a solution of $\mathbf{4}(0.268 \mathrm{~g}, 1 \mathrm{mmol})$ in toluene $(30 \mathrm{~mL})$ at room temperature. The mixture was stirred and heated to reflux for $5 \mathrm{~h}$. The reaction mixture was filtered while it was still hot. The solvents were removed on a rotary evaporator at $40^{\circ} \mathrm{C}$. The residue was washed with ether $(3 \times 15 \mathrm{~mL})$. The product was crystallized from toluene and dried in vacuo at $70^{\circ} \mathrm{C}$. Yield: $0.18 \mathrm{~g}(62 \%) ; \mathrm{mp}$ $147^{\circ} \mathrm{C}$; IR $\left(v, \mathrm{~cm}^{-1}\right)$ : $3314(\mathrm{NH}), 3068(\mathrm{Ar} \mathrm{CH}), 2934$ (aliphatic $\mathrm{CH}), 1649(\mathrm{C}=\mathrm{O}$, amide), 1614-1486 ( $\mathrm{C}=\mathrm{C}$ and $\mathrm{C}=\mathrm{N})$; ${ }^{1} \mathrm{H} \mathrm{NMR}\left(300 \mathrm{MHz}, \mathrm{CDCl}_{3}\right.$ ) $\delta$ (ppm): 9.47 (d, J=8.62 Hz, $1 \mathrm{H}$, Ar-H), 8.60-8.57 (m, 2H, Ar-H), 8.34 (br, s, 1H, NH), 8.12 $(\mathrm{d}, \mathrm{J}=8.53 \mathrm{~Hz}, 1 \mathrm{H}, \mathrm{Ar}-\mathrm{H}), 7.94(\mathrm{t}, \mathrm{J}=7.71,1 \mathrm{H}, \mathrm{Ar}-\mathrm{H}), 7.67$ (t, $\mathrm{J}=7.55,1 \mathrm{H}, \mathrm{Ar}-\mathrm{H}), 7.60-7.53(\mathrm{~m}, 3 \mathrm{H}$, other $\mathrm{Ar}-\mathrm{H}), 3.55$ (q, $\mathrm{J}=7.11,2 \mathrm{H}, \mathrm{NHCH}_{2}$ ), 1.77 (hextet, J=7.22 Hz, 2H, $\underline{\mathrm{C}}_{2} \mathrm{CH}_{3}$ ),
1.08 (t, J=7.35 Hz, 3H, $\left.\underline{\mathrm{C}}_{3}\right) ;{ }^{13} \mathrm{C} \mathrm{NMR}\left(75 \mathrm{MHz}, \mathrm{CDCl}_{3}\right.$ ) $\delta$ (ppm): 164.87 ( $\mathrm{C}=\mathrm{O}$, amide), 158.79, 155.75, 153.21, 137.25, 134.38, 130.85, 128.78, 128.69, 128.35, 127.77, $121.11,41.33\left(\mathrm{NHCH}_{2}\right), 22.91\left(\mathrm{CH}_{2} \mathrm{CH}_{3}\right), 11.56\left(\mathrm{CH}_{2} \mathrm{CH}_{3}\right)$; HRMS (QTOF-ESI): $\mathrm{m} / \mathrm{z}$ calcd for $\mathrm{C}_{18} \mathrm{H}_{17} \mathrm{~N}_{3} \mathrm{O}: 291.1372$ found: $292.1435[M+1]^{+}$.

\section{2-Phenyl-quinazoline-4-carboxylic acid isopropy-} lamide (5c). İsopropylamine $(0.118 \mathrm{~g}, 2 \mathrm{mmol})$ was added to a solution of $4(0.268 \mathrm{~g}, 1 \mathrm{mmol})$ in toluene $(30 \mathrm{~mL})$ at room temperature. The mixture was stirred and heated to reflux for $5 \mathrm{~h}$. The reaction mixture was filtered while it was still hot. The solvents were removed on a rotary evaporator at $40^{\circ} \mathrm{C}$. The residue was washed with ether $(3 \times 15 \mathrm{~mL})$. The product was crystallized from toluene-hexane mixture and dried in vacuo at $70^{\circ} \mathrm{C}$. Yield: $0.17 \mathrm{~g}(58 \%) ; \mathrm{mp} 143^{\circ} \mathrm{C}$; IR $\left(v, \mathrm{~cm}^{-1}\right)$ : 3300 (NH), 3067 (Ar CH), 2936 (aliphatic CH), 1642 (C=O, amide), 1613-1486 ( $\mathrm{C}=\mathrm{C}$ and $\mathrm{C}=\mathrm{N}) ;{ }^{1} \mathrm{H}$ NMR $(300 \mathrm{MHz}$, $\left.\mathrm{CDCl}_{3}\right) \delta$ (ppm): 9.47 (d, J=8.30 Hz, $\left.1 \mathrm{H}, \mathrm{Ar}-\mathrm{H}\right), 8.60-8.55$ $(\mathrm{m}, 2 \mathrm{H}, \mathrm{Ar}-\mathrm{H}), 8.13-8.08(\mathrm{~m}, 2 \mathrm{H}, \mathrm{NH}$ and $\mathrm{Ar}-\mathrm{H}), 7.93(\mathrm{t}$, $\mathrm{J}=7.67,1 \mathrm{H}, \mathrm{Ar}-\mathrm{H}), 7.67(\mathrm{t}, \mathrm{J}=7.86,1 \mathrm{H}, \mathrm{Ar}-\mathrm{H}), 7.61-7.52(\mathrm{~m}$, $3 \mathrm{H}$, other $\mathrm{Ar}-\mathrm{H}$ ), 4.37 (octet, J=5.10, $1 \mathrm{H}, \mathrm{NHC} \underline{\mathrm{H}}$ ), 1.39 (d, $\left.\mathrm{J}=6.57 \mathrm{~Hz}, 6 \mathrm{H}, 2 \mathrm{CH}_{3}\right) ;{ }^{13} \mathrm{C} \mathrm{NMR}\left(75 \mathrm{MHz}, \mathrm{CDCl}_{3}\right) \delta$ (ppm): 165.54 ( $\mathrm{C}=\mathrm{O}$, amide), 160.37, 155.83, 153.17, 137.24, 134.35, 130.85, 128.77, 128.69, 128.36, 128.30, 127.75, 121.11, $41.72(\mathrm{CH}), 22.72\left(\mathrm{CH}_{3}\right)$; HRMS (QTOF-ESI): $\mathrm{m} / \mathrm{z}$ calcd for $\mathrm{C}_{18} \mathrm{H}_{17} \mathrm{~N}_{3} \mathrm{O}$ : 291.1372; found: $292.1447[\mathrm{M}+1]^{+}$.

12. 2-Phenyl-quinazoline-4-carboxylic acid phenylamide (5d). Aniline (0.186 g, $2 \mathrm{mmol}$ ) was added to a solution of 4 ( $0.268 \mathrm{~g}, 1 \mathrm{mmol})$ in THF $(30 \mathrm{~mL})$ at room temperature. The mixture was stirred and heated to reflux for $6 \mathrm{~h}$. The reaction mixture was filtered while it was still hot. The solvents were removed on a rotary evaporator at $40^{\circ} \mathrm{C}$. The residue was washed with ether $(3 \times 15 \mathrm{~mL})$. The product was crystallized from THF-hexane mixture and dried in vacuo at $40^{\circ} \mathrm{C}$. Yield: $0.17 \mathrm{~g}(52 \%) ; \mathrm{mp} 117^{\circ} \mathrm{C}$; IR ( $\left.\mathrm{v}, \mathrm{cm}^{-1}\right)$ : $3315(\mathrm{NH}), 3063$ ( $\mathrm{Ar}$ $\mathrm{CH}), 1663(\mathrm{C}=\mathrm{O}$, amide), 1614-1486 (C=C and $\mathrm{C}=\mathrm{N}) ;{ }^{1} \mathrm{H}$

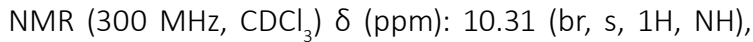
$9.57(\mathrm{~d}, \mathrm{~J}=8.64 \mathrm{~Hz}, 1 \mathrm{H}, \mathrm{Ar}-\mathrm{H}), 9.42$ (d, J=8.61 Hz, 1H, Ar$H), 8.64-8.56(m, 3 H, A r-H), 8.22-8.16(m, 2 H, A r-H), 7.86$ (d, J=7.53 Hz, 2H, Ar-H), 7.60-7.57 (m, 4H, Ar-H), 7.49 (t, $\mathrm{J}=7.53 \mathrm{~Hz}, 1 \mathrm{H}, \mathrm{Ar}-\mathrm{H}) ;{ }^{13} \mathrm{C} \mathrm{NMR}\left(75 \mathrm{MHz}, \mathrm{CDCl}_{3}\right) \delta(\mathrm{ppm})$ : 164.17 ( $C=O$, amide), 162.46, 158.57, 153.46, 152.43, 137.30, 134.63, 131.02, 129.22, 129.00, 128.93, 128.66, 128.35, 127.61, 124.98, 120.13, 116.23; HRMS (QTOFESI): $\mathrm{m} / \mathrm{z}$ calcd for $\mathrm{C}_{21} \mathrm{H}_{15} \mathrm{~N}_{3} \mathrm{O}$ : 325.1215; found: 325.1454 $[\mathrm{M}]^{+}$. 
13. 2-Phenyl-quinazoline-4-carboxylic acid (4-sulfamoyl-phenyl)-amide (5e). 4-aminobenzenesulfonamide ( $0.344 \mathrm{~g}, 2 \mathrm{mmol})$ was added to a solution of $4(0.268 \mathrm{~g}, 1 \mathrm{mmol})$ in THF $(30 \mathrm{~mL})$ at room temperature. The mixture was stirred and heated to reflux for $6 \mathrm{~h}$. The reaction mixture was filtered while it was still hot. The solvents were removed on a rotary evaporator at $40^{\circ} \mathrm{C}$. The residue was washed with ether $(3 \times 15 \mathrm{~mL})$. The product was crystallized from THF-toluene mixture and dried in vacuo at $70^{\circ} \mathrm{C}$. Yield: $0.18 \mathrm{~g}(44 \%) ; \mathrm{mp}$ $285^{\circ} \mathrm{C}$; IR ( $\left.v, \mathrm{~cm}^{-1}\right)$ : $3331(\mathrm{NH}), 3105(\mathrm{Ar} \mathrm{CH}), 1681$ (C=O, amide), 1615-1485 ( $\mathrm{C}=\mathrm{C}$ and $\mathrm{C}=\mathrm{N}), 1342\left(\mathrm{SO}_{2}\right.$ asym.), 1155 ( $\mathrm{SO}_{2}$ sym.); ${ }^{1} \mathrm{H}$ NMR (300 MHz, DMSO-d $\left.{ }_{6}\right) \delta$ (ppm): $11.35(\mathrm{~s}, 1 \mathrm{H}, \mathrm{NH}), 8.70-8.61(\mathrm{~m}, 3 \mathrm{H}, \mathrm{Ar}-\mathrm{H}), 8.22-8.05(\mathrm{~m}$, $4 \mathrm{H}, \mathrm{Ar}-\mathrm{H}), 7.91-7.80(\mathrm{~m}, 3 \mathrm{H}, \mathrm{Ar}-\mathrm{H}), 7.63-7.61(\mathrm{~m}, 3 \mathrm{H}$, other Ar-H), 7.36 (s, 2H, SO $\left.\mathrm{NH}_{2}\right) ;{ }^{13} \mathrm{C}$ NMR (75 MHz, DMSO-d $\mathrm{d}_{6}$ ) $\delta$ (ppm): 164.30 (C=O, amide), 160.28, 159.29, 152.12, $141.55,140.13,137.13,135.85,131.74,129.30,129.13$ $128.95,128.23,127.23,126.76,120.65,120.12$; HRMS (QTOF-ESI): $\mathrm{m} / \mathrm{z}$ calcd for $\mathrm{C}_{21} \mathrm{H}_{16} \mathrm{~N}_{4} \mathrm{O}_{3} \mathrm{~S}$ : 404.0943; found: $405.1014[\mathrm{M}+1]^{+}$.

\section{RESULTS and DISCUSSION}

When we look at the literature, it is seen in our study that we synthesize the carboxylic acid derivative which is directly bound to the quinazoline ring for the first time. Because the quinazoline derivatives show a wide range of biological activities, we hope that the compounds we synthesized in this study will contribute to medical chemistry.

When we look at the reaction mechanism, an aromatic amine compound (DMAP) is used as a catalyst in the synthesis of quinazoline. Since we do not use a catalyst in our reaction, it is believed that the catalytic function is the hydrolysis isatin compound containing the aromatic amine group. That is, the hydrolysis isatin compound acts as both a catalyst and a component of the reaction. At the same time, while the hydrolysis isatin compound is insoluble in hot ethanol, it completely dissolves in warm ethanol together with ammonium acetate and benzaldehyde. The quinazoline derivative carboxyl acid salt formed in the hot ethanol during the reaction forms a precipitate. This allowed the reaction to be separated from the other reaction components and by-products by hot filtration. In the one-pot three-component reaction in the quinazoline synthesis, the reaction time was very long (24 h) and the yield (29\%) was very low, but we could easily distinguish it from the reaction medium.
Carboxylic acid ester derivatives were readily obtained in the presence of sulfuric acid by the reaction of alkyl alcohols and the quinazoline carboxylic acid compound. However, the phenol ester can be obtained by reacting the phenol with the carboxylic acid chloride which is more active than the carboxylic acid compound. Similarly, quinazoline amide derivatives were also obtained from the simple reaction of the carboxylic acid chloride compound with various amines.

As a result, we successfully synthesized ester and amide derivatives of the quinazoline-4-carboxylic acid compound we synthesized by one-pot three-component reaction. Since the quinazoline compounds exhibit versatile biological activity, we think that the compounds we synthesize will contribute to medicinal chemistry.

\section{Acknowledgements}

We are grateful to the Dumlupinar University Technology Research Fund for the financial support of this study through project

number of $2015 / 24$.

\section{References}

1. H.M. Shallal, W.A. Russu, Discovery, synthesis, and investigation of the antitumor activity of novel piperazinylpyrimidine derivatives, Eur. J. Med. Chem., 46 (2011) 2043-2057.

2. G. Grover, S.G. Kini, Synthesis and evaluation of new quinazolone derivatives of nalidixic acid as potential antibacterial and antifungal agents, Eur. J. Med. Chem., 41 (2006) 256-262.

3. A.B.A. El-Gazzar, M.M. Youssef, A.M.S. Youssef, A.A. Abu-Hashem, F.A. Badria, Design and synthesis of azolopyrimidoquinolines, pyrimidoquinazolines as antioxidant, anti-inflammatory and analgesic activities, Eur. J. Med. Chem., 44 (2009) 609-624.

4. P. Verhaeghe, N. Azas, M. Gasquet, S. Hutter, C. Ducros, M. Laget, S. Rault, P. Rathelot, P. Vanelle, Synthesis and antiplasmodial activity of new 4-aryl-2trichloromethylquinazolines, Bioorg. Med. Chem. Lett., 18 (2008) 396-401.

5. R.A. Smits, M. Adami, E.P. Istyastono, O.P. Zuiderveld, C.M.E. van Dam, F.J.J. de Kanter, A. Jongejan, G. Coruzzi, R. Leurs, I.J.P. de Esch, Synthesis and QSAR of quinazoline sulfonamides as highly potent human histamine $\mathrm{h}-4$ receptor inverse agonists, J. Med. Chem., 53 (2010) 2390-2400.

6. C. Mendoza-Martinez, J. Correa-Basurto, R. NietoMeneses, A. Marquez-Navarro, R. Aguilar-Suarez, M.D. Montero-Cortes, B. Nogueda-Torres, E. Suarez-Contreras, N. Galindo-Sevilla, A. Rojas-Rojas, A. Rodriguez-Lezama, F. Hernandez-Luis, Design, synthesis and biological evaluation of quinazoline derivatives as anti-trypanosomatid and antiplasmodial agents, Eur. J. Med. Chem., 96 (2015) 296-307. 
7. T. Herget, M. Freitag, M. Morbitzer, R. Kupfer, T. Stamminger M. Marschall, Novel chemical class of pUL97 protein kinasespecific inhibitors with strong anticytomegaloviral activity, Antimicrob. Agents. Ch., 48 (2004) 4154-4162.

8. T.P. Selvam, P.V. Kumar, A.S. Kumar, I.A. Emerson, Study of inhibitory mechanism and binding mode of the thiazolo quinazoline compounds to HIV-1 integrase by docking, J. Pharm. Res., 3 (2010) 1637-1647.

9. S.K. Kashaw, V. Kashaw, P. Mishra, N.K. Jain, J.P. Stables, Synthesis, anticonvulsant and CNS depressant activity of some new bioactive 1-(4-substituted-phenyl)-3-(4-oxo-2 phenyl/ethyl-4H-quinazolin-3-yl)-urea, Eur. J. Med. Chem., 44 (2009) 4335-4343.

10. M.S. Malamas, J. Millen, Quinazolineacetic acids and related analogs as aldose reductase inhibitors, J. Med. Chem., 34 (1991) 1492-1503.

11. J.P. Xu, X.Y. Liu, S. Yang, C.G. Zhang, L. Wang, Y.K. Shi, Icotinib as initial treatment in lung adenocarcinoma patients with brain metastases, Thorac. Cancer, 7 (2016) 437-441.

12. D. Cameron, M. Casey, M. Press, D. Lindquist, T. Pienkowski, C.G. Romieu, S. Chan, A. Jagiello-Gruszfeld, B. Kaufman, J. Crown, A. Chan, M. Campone, P. Viens, N. Davidson, V. Gorbounova, J.I. Raats, D. Skarlos, B. Newstat, D. Roychowdhury, P. Paoletti, C. Oliva, S. Rubin, S. Stein, C.E. Geyer, A phase III randomized comparison of lapatinib plus capecitabine versus capecitabine alone in women with advanced breast cancer that has progressed on trastuzumab: updated efficacy and biomarker analyses, Breast Cancer Res. Treat., 112 (2008) 533-543.

13. R.N. Brogden, R.C. Heel, T.M. Speight, G.S. Avery, Prazosin: a review of its pharmacological properties and therapeutic efficacy in hypertension, Drugs, 14 (1977) 163-197.

14. R.S. Herbst, Erlotinib (Tarceva): an update on the clinical trial program, Semin. Oncol., 30 (2003) 34-46.

15. M. Murphy, B. Stordal, Erlotinib or gefitinib for the treatment of relapsed platinum pretreated non-small cell lung cancer and ovarian cancer: a systematic review, Drug Resist. Updat., 14 (2011) 177-190.

16. G.M. Chinigo, M. Paige, S. Grindrod, E. Hamel, S. Dakshanamurthy, M. Chruszcz, W. Minor, M.L. Brown Asymmetric synthesis of 2,3-dihydro-2-arylquinazolin-4ones: methodology and application to a potent fluorescent tubulin inhibitor with anticancer activity, J. Med. Chem., 51 (2008) 4620-4631.

17. T. Sardon, T. Cottin, J. Xu, A. Giannis, I. Vernos, Development and biological evaluation of a novel aurora $A$ kinase inhibitor, Chembiochem., 10 (2009) 464-478.

18. S.L. Cao, Y. Wang, L. Zhu, J. Liao, Y.W. Guo, L.L. Chen, H.Q $\mathrm{Liu}, \mathrm{X} . \mathrm{Xu}$, Synthesis and cytotoxic activity of N-((2-methyl4(3H)-quinazolinon-6-yl)methyl)dithiocarbamates, Eur. J. Med. Chem., 45 (2010) 3850-3857.
19. D.W. Fry, A.J. Kraker, A. McMichael, L.A. Ambroso, J.M. Nelson, W.R. Leopold, R.W. Connors, A.J. Bridges, A specific inhibitor of the epidermal growth factor receptor tyrosine kinase, Science, 265 (1994) 1093-1095.

20. N. Malecki, P. Carato, B. Rigo, J.F. Goossens, R. Houssin, C. Bailly, J.P. Henichart, Synthesis of condensed quinolines and quinazolines as DNA ligands, Bioorgan. Med. Chem., 12 (2004) 641-647.

21. H.N. Seo, J.Y. Choi, Y.J. Choe, Y. Kim, H. Rhim, S.H. Lee, J. Kim, D.J. Joo, J.Y. Lee, Discovery of potent T-type calcium channel blocker, Bioorg. Med. Chem. Lett., 17 (2007) 5740-5743.

22. W. Zhang, F. Guo, F. Wang, N. Zhao, L. Liu, J. Li, Z.H. Wang, Synthesis of quinazolines via $\mathrm{CuO}$ nanoparticles catalyzed aerobic oxidative coupling of aromatic alcohols and amidines, Org. Biomol. Chem., 12 (2014) 5752-5756.

23. C. Huang, Y. Fu, H. Fu, Y.Y. Jiang, Y.F. Zhao, Highly efficient copper-catalyzed cascade synthesis of quinazoline and quinazolinone derivatives, Chem. Commun., (2008) 63336335.

24. C.C. Malakar, A. Baskakova, J. Conrad, U. Beifuss, CopperCatalyzed Synthesis of Quinazolines in Water Starting from o-Bromobenzylbromides and Benzamidines, Chem. Eur. J., 18 (2012) 8882-8885.

25. R. Alonso, A. Caballero, P.J. Campos, D. Sampedro, M.A. Rodriguez, An efficient synthesis of quinazolines: a theoretical and experimental study on the photochemistry of oxime derivatives, Tetrahedron, 66 (2010) 4469-4473.

26. Y. Ohta, Y. Tokimizu, S. Oishi, N. Fujii, H. Ohno, Direct Synthesis of Quinazolines through Copper-Catalyzed Reaction of Aniline-Derived Benzamidines, Org. Lett., 12 (2010) 3963-3965

27. Z.H. Zhang, X.N. Zhang, L.P. Mo, Y.X. Li, F.P. Ma, Catalyst-free synthesis of quinazoline derivatives using low melting sugarurea-salt mixture as a solvent, Green Chem., 14 (2012) 15021506.

28. F. Portela-Cubillo, J.S. Scott, J.C. Walton, MicrowavePromoted syntheses of quinazolines and dihydroquinazolines from 2-aminoarylalkanone o-phenyl oximes, J. Org. Chem., 74 (2009) 4934-4942.

29. J.T. Zhang, D.P. Zhu, C.M. Yu, C.F. Wan, Z.Y. Wang, A simple and efficient approach to the synthesis of 2-phenylquinazolines via sp(3) C-H functionalization, Org. Lett., 12 (2010) 28412843

30. C. Derabli, R. Boulcina, G. Kirsch, B. Carboni, A. Debache, A DMAP-catalyzed mild and efficient synthesis of 1,2-dihydroquinazolines via a one-pot three-component protocol, Tetrahedron Lett., 55 (2014) 200-204. 